$$
\text { No. } 4109 \text { July 31, } 1948
$$

to test it for possible carcinogenicity (see following letter).

Department of Zoology,

LL. LLOYD

University, Leeds 2.

${ }^{1}$ Mottram, J. C., Cancer Res., 1, 313 (1941).

'Mottram, J. C., "Problem of Tumours" (London, 1942).

${ }^{3}$ Calkins, G. N., Biol. Bull., 21, 36 (1911).

- Peebles, F., Biol. Bull., 23, 154 (1912).

' Chatton, E., C.R. Acad. Sci., Paris, 173, 393 (1921).

- de Garis, C. F., J. Exp. Zool., 49, 133 (1927).

"Lloyd, Ll., Nature, 159, 135 (1947).

${ }^{8}$ Herzfeld, E., Jen. Z. Natur., 62, 79 (1926).

- Stocking, R. J., J. Exp. Zool., 19, 387 (1915).

${ }^{10}$ Jennings, H. S., J. Exp. Zool., 5, 577 (1908).

\section{Absence of Carcinogenic Activity of Benzene Hexachloride ('Gammexane')}

For reasons given in the preceding letter, benzene hexachloride has been tested for carcinogenic pro. perties. A 0.5 per cent solution in acetone has been applied twice weekly for fifteen months to the interscapular skin of a group of thirty mixed stock mice. A further twenty mice were subcutaneously implanted with pellets of paraffin wax containing 3 per cent of 'Gammexane', and have since been observed for ten months.

No tumours have been induced in either group. Moreover, there have been none of the changes seen with known carcinogens during the latent period, for example, epilation, local thickening and congestion of the skin, epidermal hyperplasia, and fibrillary changes in the dermis of the first group ${ }^{1}$; or the characteristic failure to form a firm collagenous capsule round the pellet in the second group ${ }^{2}$. The survival-rate has been satisfactory, twenty-one and twelve out of the original thirty and twenty respect. ively being still alive at the time of writing. Of the dead mice, one showed leukæmia at autopsy and another was killed following the observation of a carcinoma of the breast; both these lesions occur spontaneously in the stock, and there is no reason to attribute them to the treatment.

It is perhaps worthy of mention that the general condition of the mice in the first group has been definitely superior to that obtaining in an average experiment; this we attribute to the reduction of parasitic cutaneous infestation. In certain types of prolonged experiment, periodical treatment of the animals in this way might prove of general value.

Department of Experimental Pathology, University, Leeds 2.

${ }^{1}$ Orr, J. W., J. Path. Bact., 46, 495 (1938).

'Orr, J. W., J. Path. Bact., 47, 157 (1939).

\section{Rhythmic Respiration in the Sea Urchin}

I AM much interested in the note by Dr. Erik Zeuthen on the presence of rhythmic respiration in sea urchin eggs ${ }^{1}$. Fifteen years ago, while at Harvard, I performed a number of experiments on the fertilized eggs of another species of the sea urchin, Arbacia punctulata, using the Warburg microrespirometer technique. I found rhythmic variation in the rate of oxygen consumption by these more or less uniformly cleaving eggs. The phenomenon is quite pronounced at $25^{\circ}$, but not so at $15^{\circ}$. The oxygen consumption $v$. time, or oxygen consumption $v$. cleavage curves are very much the same as those given by Dr. Zeuthen. I also discussed in detail the reasons for the failure of previous workers to find such variations. In Warburg's first experiments ${ }^{2}$ the readings were taken at too long intervals, and Gray's ${ }^{3}$ experiments were made at too low a temperature $\left(11^{\circ}\right)$ for the variations to be significant. Shearer's data actually showed such a variation if his data were replotted differentially as rate against time, rather than as an integral curve of amount of oxygen consumed against time, which was the way he plotted the results.

The results of my experiments, together with the discussion, were published, upon my return to China, in Wuhan University Journal of Science, 7, 1 (1937). Unfortunately, war broke out soon after, and very few copies of the journal are in existence now. Even I myself have no copy. I am trying to locate this article, both in China and in libraries abroad.

Physiological Laboratory, Peisung Tang

Tsing Hua University, Peiping.

March 5.

${ }^{1}$ Nature, 160, 577 (1947)

2 Warburg, O., "Stoffwechsel der Tumoren" (Berlin, 1926).

${ }^{3}$ Gray, J., Proc. Phil. Soc., Biol. Sect., 1, 164 (1924).

\section{Use of Rotifera for Testing Analgesics, Narcotics and Similar Drugs}

THE assay of the analgesic power of therapeutic substances by means of rats cannot be carried out with any degree of precision. It has been found that the Rotifera can be employed for this purpose since they are extremely sensitive to killing agents unless they are previously narcotized ${ }^{1}$.

Through the courtesy and active help of Mr. E. D. Hollowday, regular supplies of the rotifer, Brachionis calyciflorus, were obtained, and lots of $1.0 \mathrm{ml}$. of the water containing 10-15 of the Rotifera pipetted into small tubes. An equal volume of a solution of the drug under test at a known concentration was slowly added, the tube being gently rotated to ensure even mixing. After allowing to stand for $10 \mathrm{~min}$. the Rotifera were examined under the microscope. After noting their condition, the animalcules were killed with formalin and the state of the cilia determined.

The various states were defined as follows :

(i) Dead. These had died from 'natural' causes and generally consisted of the lorica containing a more or less decomposed internal mass.

(ii) Killed. These had contracted on contact with the formalin or other drug without passing through a state of narcosis. This was indicated by contraction of the cilia, generally accompanied by contortion of the internal organs.

(iii) Narcotized. These showed no contraction or contortion, the Rotifera being free from movement but with the cilia outstretched. On the addition of formalin, no immediate obvious changes took place.

(iv) Alive but not narcotized. These were either static or else free-swimming with the normal movement of the cilia. On the addition of formalin, they contracted and contorted within the lorica.

The relative numbers in the three classes, killed, narcotized, and alive but not narcotized, were counted.

The effect of various drugs was examined throughout ranges of concentration depending on the activity of the particular substance under investigation. The 